\title{
Risk Factors for Pseudarthrosis in Minimally- Invasive Transforaminal Lumbar Interbody Fusion
}

\author{
Arash Emami, Michael Faloon, Nikhil Sahai, Conor J. Dunn, Kimona Issa, \\ Daniel Thibaudeau, Kumar Sinha, Ki Soo Hwang \\ Department of Orthopaedic Surgery, Seton Hall University School of Health and Medical Sciences, South Orange, NJ, USA
}

\begin{abstract}
Study Design: Retrospective cohort study (level of evidence: 4).
Purpose: To describe the potential comorbid, operative, and radiographic risk factors for the development of clinically-relevant pseudarthrosis following minimally-invasive transforaminal lumbar interbody fusion (MIS-TLIF).

Overview of Literature: MIS-TLIF has shown long-term clinical outcomes with decreased perioperative morbidity and earlier return to work, similar to those of open TLIF. However, unsuccessful fusion still remains a concern. The impacts of various patient, operative, and radiographic risk factors have not been evaluated for their potential association with pseudarthrosis related to MIS-TLIF.

Methods: Between 2012 and 2015, 204 consecutive patients underwent one or two-level MIS-TLIF at St. Joseph's University Medical Center, Paterson, NJ, USA; they had a minimum of 1 year of follow-up. The patients were divided into two cohorts: those who developed clinically-relevant pseudarthrosis and those who did not. Clinically-relevant pseudarthrosis was determined by both evidence on computed tomography and presence of continued clinical symptoms at 1-year follow-up.

Results: Revision surgery was the only identified non-radiographic factor associated with pseudarthrosis. Disc angle had the highest $\left(R^{2}=0.8\right)$, followed by anterior disc height $\left(R^{2}=0.79\right)$. Although posterior disc height and the ratio of anterior to posterior disc height showed a marked relationship with the outcome, the $R^{2}$-values were $<0.3$, thus indicating a less-strong correlation. The overall pseudarthrosis rate was $8 \%$. No statistically significant differences were identified between the two cohorts with respect to mean age, sex, medical comorbidities, smoking status, or number of levels fused.

Conclusions: Clinically-relevant pseudarthrosis is not uncommon following MIS-TLIF. In the current study, undergoing revision surgery, disc angle, and anterior disc height were observed to be associated with clinically-relevant pseudarthrosis. This study demonstrated that the patient population may benefit from an alternate approach.
\end{abstract}

Keywords: Pseudarthrosis; Minimally-invasive transforaminal lumbar interbody fusion; Radiographic findings; Disc height; Disc angle; Revision surgery

\section{Introduction}

Transforaminal lumbar interbody fusion (TLIF) has traditionally been safe and effective for treating degenerative lumbosacral diseases of the spine [1]. It was developed as an alternative procedure to, and as a means of addressing some of the challenges associated with, posterior lumbar interbody fusion (PLIF), including thecal sac retraction and narrow exposure to the interbody space. Recently, minimally-invasive techniques have been applied to the

Received Oct 19, 2017; Revised Dec 29, 2017; Accepted Jan 31, 2018

Corresponding author: Michael Faloon

University Spine Center, 504 Valley Road, Suite 203, Wayne, NJ 07470, USA

Tel: +1-973-686-0700 (x199), Fax: +1-973-686-0701, E-mail: faloonmi@gmail.com 
traditional "open" TLIF to reduce stripping of the paravertebral muscles, lessen damage to posterior elements, and decrease postoperative pain, time to ambulation, postoperative narcotic use, intraoperative blood loss, and wound drainage with the ultimate goal of achieving a shorter hospital course and quicker return to work. However, recent evidence suggests that minimally-invasive TLIF (MIS-TLIF) has outcomes comparable with those of open TLIF; thus, pseudarthrosis, or nonunion of the fusion, still continues to be a problem [2-7].

Pseudarthrosis in spine surgery was first classified by Heggeness and Esses [8] in 1991. Posterolateral pseudarthrosis of the lumbar spine results from failure of bone fusion, is typically evident at 1 year after attempted spinal fusion, and is classified into four types: transverse, atrophic, shingle, and complex [8-11]. Fusion rates are reportedly influenced by several variables, including modifiable patient behaviors, such as nutritional status, smoking/ nicotine intake, diabetes mellitus, rheumatoid arthritis, non-steroidal anti-inflammatory drugs, and corticosteroids [12-15]. In addition, fusion success may also be altered by several operative factors, including number of levels fused, use of instrumentation, bone graft selection, augmentation with biologics, surgical technique, proper sizing, and choice of interbody cages, among others $[9,12]$.

It remains unclear whether minimally-invasive applications in a large cohort improve the rate of clinicallyrelevant pseudarthrosis (defined as confirmed pseudarthrosis on computed tomography (CT) with persistent symptomatology). There is also a lack of literature examining clinical and radiographic risk factors related to the development of pseudarthrosis following MIS-TLIF. Thus, we specifically compared the following: (1) baseline patient demographics, comorbidities, and number of spinal fusion levels; (2) preoperative radiographic findings; (3) clinical outcomes, including the Oswestry Disability Index (ODI) score, and (4) complication rates between patients undergoing MIS-TLIF with and without clinicallyrelevant pseudarthrosis.

\section{Materials and Methods}

This study was exempt from Institutional Review Board approval and informed consent was waived for this retrospective chart review. A retrospective review of our institution's database (2012-2015) yielded 204 consecutive patients who underwent one- or two-level minimally- invasive TLIF with a bilateral screw and rod construction. Patients with complete medical records and minimum 1-year follow-up were included. Patients were divided into two cohorts for risk factor analysis: those who experienced pseudarthrosis and those who did not. Only those patients having accessible preoperative X-rays were further included for radiographic parameter analysis. Charlson Comorbidity Scores (with age scores included) were calculated for each patient [16].

All surgical procedures were performed at a single institution between 2012 and 2015. Indications for surgery included degenerative disc disease or lumbar spinal stenosis with spondylolisthesis (grades 1 and 2). Additionally, patients undergoing surgery for recurrent stenosis, recurrent disc herniation, prior herniated disc decompression, radiculopathy requiring complete facetectomy, and iatrogenic instability were included. Patients with previously performed fusion procedures at the operative level(s) were excluded. All medical information, including history and physical examinations, operative notes, discharge summaries, follow-up office visits, and magnetic resonance imaging (MRIs), was carefully reviewed.

All patients were clinically assessed at 2 weeks postoperatively and at 6 weeks, 3 months, 6 months, and annually thereafter. The patients were thoroughly examined during each clinical visit; Visual Analog Scale back and leg pain scores and ODI scores were recorded. Demographic data, medical comorbidities, outcome scores, and imaging data were quantified. Pseudarthrosis was diagnosed if both clinical symptoms, such as recurrent radiculopathy or axial back pain, persisted and if radiographic evidence revealed by CT (Fig. 1) demonstrated unsuccessful fusion at 1-year follow-up [17]. CTs were ordered for patients who displayed the aforementioned clinical symptoms and demonstrated radiographic indications suspicious of pseudarthrosis, including increased motion across the segment, radiolucency of instrumentation, interbody cage settling, or lack of sentinel signs on lateral radiograph [18]. CT images were read by both a neuroradiologist and the attending spine surgeon, and only those patients who displayed evidence of nonunion by both physicians were included in the pseudarthrosis cohort.

\section{Operative procedure}

Each patient underwent surgery by the same technique. The side for performing surgery was determined accord- 

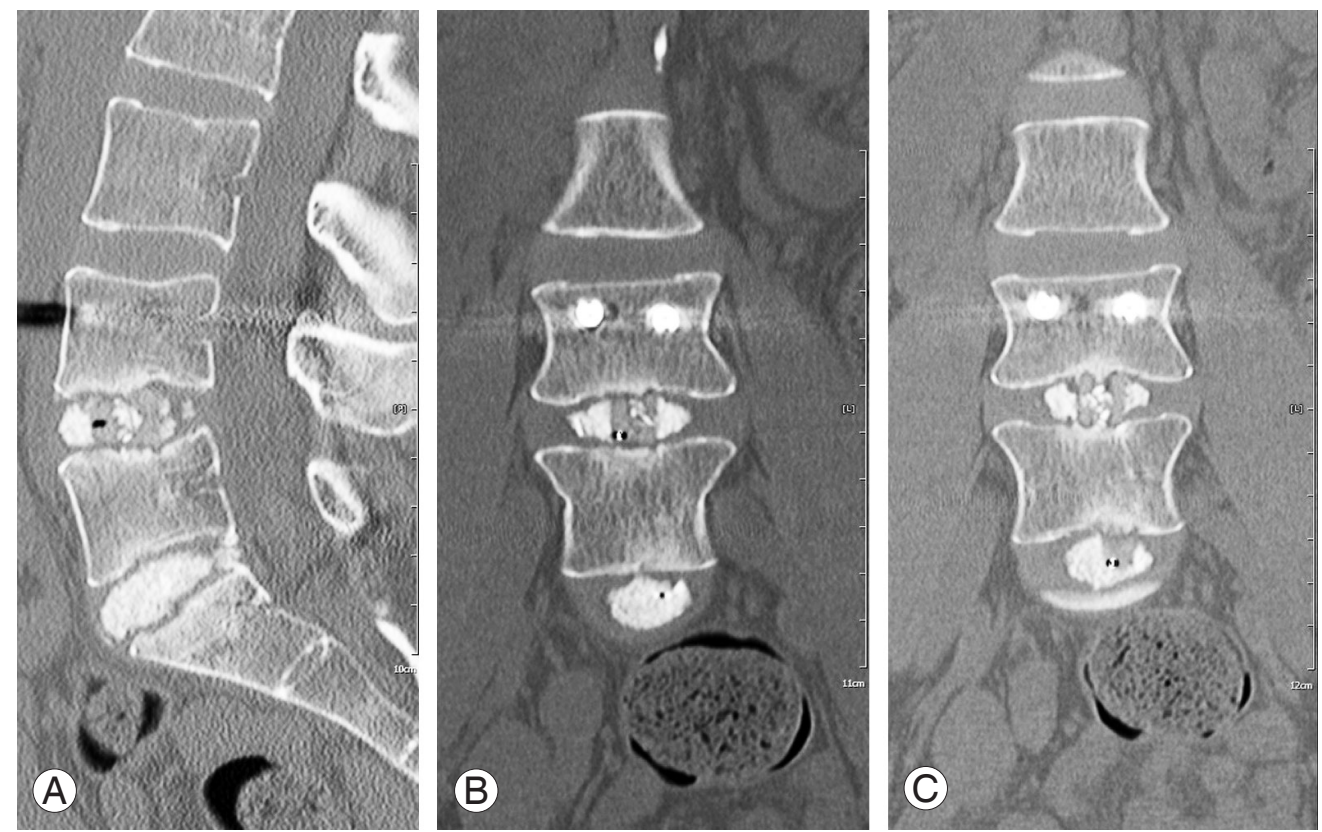

Fig. 1. (A-C) Sagittal and coronal computed tomography demonstrating pseudarthrosis at L4-5/L5-S1.

ing to the patient's symptoms. All MIS-TLIF procedures were performed by first using fluoroscopy to visualize and identify the correct levels. Care was taken to initiate a starting point to the pedicle, lateral to the facet joint at the most proximal level. Stab incisions were connected cranial-to-caudal in a paramedian or Wiltse fashion. A 24-mm docking port (Spotlight Access Port; Depuy, Raynham, MA, USA) was then inserted and docked over the facet joint after tubular retractors were used and sequential dilation of the paraspinous soft tissue was performed. Trocars were bilaterally introduced over the pedicles at the surgical levels via stab incisions. Guide wires were then bilaterally placed into the pedicles; appropriate placement was confirmed using fluoroscopy, and wires were taped to the patient. Next, a high speed burr was used to remove the inferior articular facet of the level above and the superior articular facet of the level below the site of decompression. The traversing nerve root was then identified and retracted. Annulotomy was performed after the disc space was identified.

Discectomy was performed using a series of shavers and curettes, and trial cages were then used to determine the correct size. After the disc space was filled with local bone graft (from facet/lamina) and allograft, a polyether ether ketone cage (Concorde Bullet, 7-13 $\mathrm{mm}$ and parallel to $9^{\circ}$ lordosis; Depuy) was placed. The cage size was determined on a patient-specific basis with best intraoperative fit affected by preoperative disc height. An extra-small sponge $(1.4 \mathrm{~mL})$ of ultra-low dose $(2 \mathrm{mg})$ recombinant human bone morphogenic protein-2 (rhBMP-2; Infuse, Medtronic, Minneapolis, MN, USA) was cut into several pieces and placed anterior to the cage in patients, including smokers and/or diabetics, deemed high risk for pseudarthrosis. Next, a pedicle tap was used to obtain correct screw trajectory. Pedicle screws (Viper, Depuy) were then placed, and correct positioning was confirmed via fluoroscopy. Electromyographic potential was tested across both the pedicle tap and screw with an acceptable threshold of $10 \mathrm{~mA}$. Finally, rods were placed, and the caps were applied. Pseudarthrosis was intraoperatively confirmed at the time of revision by anterior lumbar interbody fusion (ALIF).

\section{Radiographic measurement}

Radiographic measurements were performed using a standing lateral X-ray. Anterior disc height, posterior disc height, and disc angulation measurements were all performed at the surgical level. Anterior disc height was measured as the vertical distance between the anteriormost extent of both endplates surrounding the surgical level (Figs. 2, 3). Posterior disc height was measured in the same manner, but at the posterior-most extent of the surgical level. The ratio between the anterior and posterior disc 
heights was then recorded. Disc angle was measured as the angle formed between the inferior endplate above and the superior endplate below the surgical level (Figs. 4, 5).

\section{Statistical analysis}

GraphPad (2015 version; GraphPad Software Inc., San Diego, CA, USA) and R statistics (https://www.r-project. org/) was used to perform all statistical analyses. Student

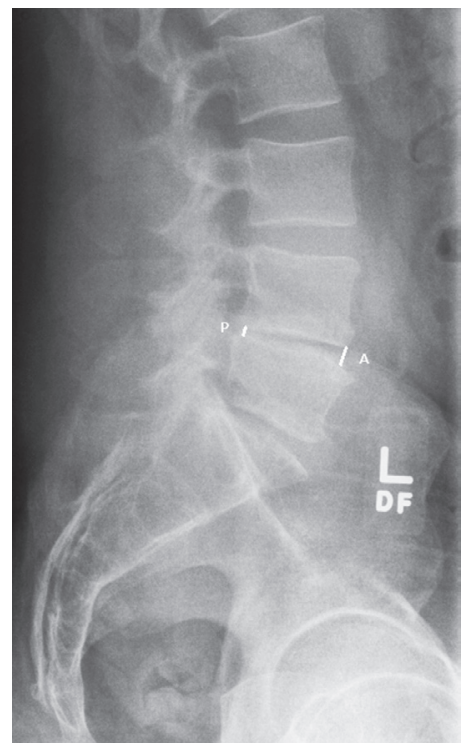

Fig. 2. Preoperative $X$-ray measurement of $L 4-L 5$ anterior and posterior disc heights in a patient who did not develop pseudarthrosis. Anterior=4.4 mm; posterior=2.9 mm; and anterior/posterior=1.5. $t$-test was used to compare patient demographics and operative factors. The original radiographic parameter analysis plan was to perform multivariate logistic regression analysis with all the significant factors in the model to determine the individual contribution of each of the four factors while also adjusting for the others. However, anterior disc height, posterior disc height, their ratio, and disc angle were highly correlated with each other. Therefore, we did not perform multivariate logistic regression analysis owing to the possible violation of the co-linearity assumption. Instead, we conducted several univariate logistic regression analyses and compared the model fit using the coefficient of determination $\left(R^{2}\right)$. The variable providing the largest $R^{2}$ was considered the most relevant factor related to a particular outcome (in this case, pseudarthrosis). All $p<0.05$ was considered statistically significant.

\section{Results}

Totally, 204 consecutive patients underwent MIS-TLIF at our institution and had complete medical records and at least 1 year of follow-up. CT and preoperative radiographs were available for 168 of the initial 204 patients. Table 1 presents the demographic characteristics of the study cohort. Totally, 16 patients were diagnosed with clinically-relevant pseudarthrosis. The pseudarthrosis cohort comprised 10 males and six females, whereas the
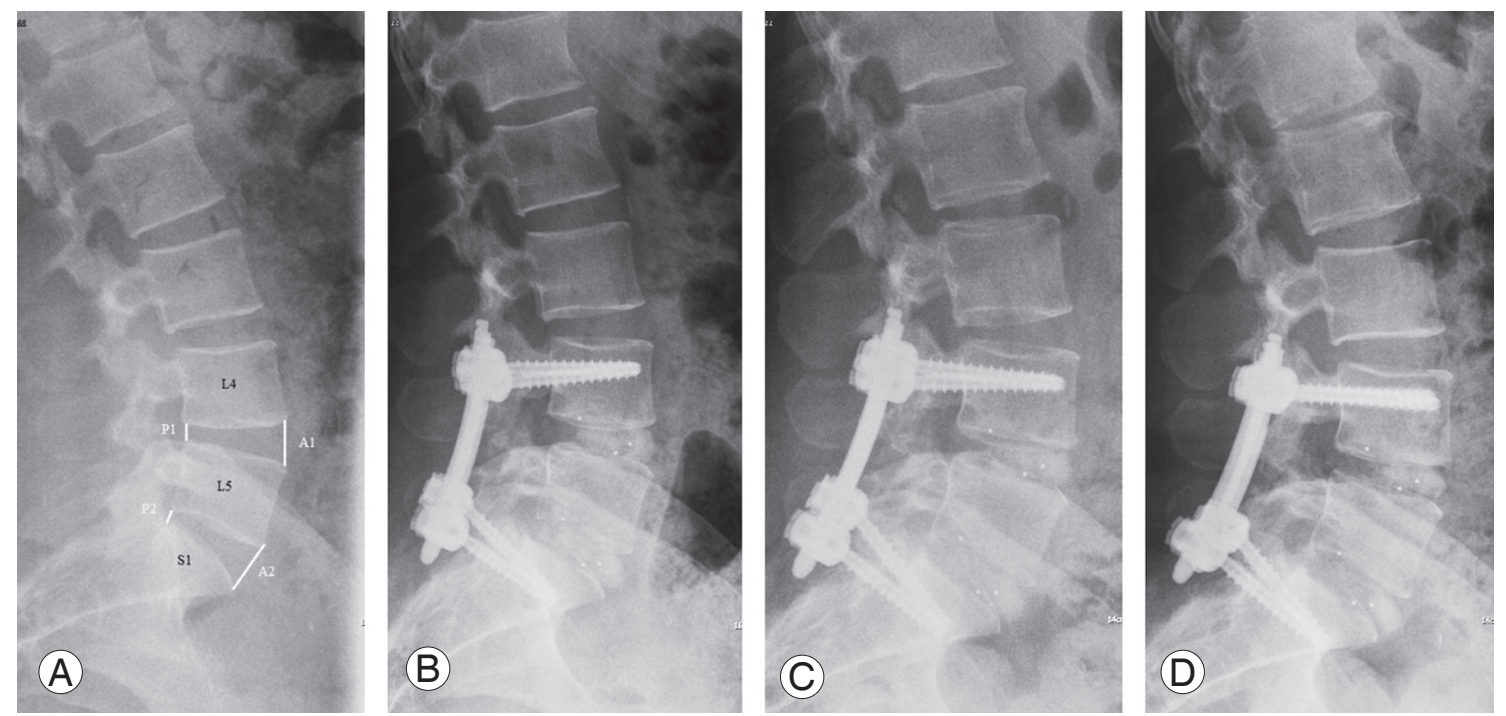

Fig. 3. Preoperative and postoperative $X$-ray measurements of anterior and posterior disc heights in a patient who developed pseudarthrosis. $\mathrm{L} 4-5$ (anterior $1=15.2 \mathrm{~mm}$ and posterior 1=6.5 mm), L5-S1 (anterior 2=18 mm and posterior 2=6 mm). Anterior $1 /$ posterior 1=2.3 and anterior 2/posterior 2=3. (A) Preoperative; (B) 6 mo postoperative; (C) 12 mo postoperative; and (D) 18 mo postoperative. 


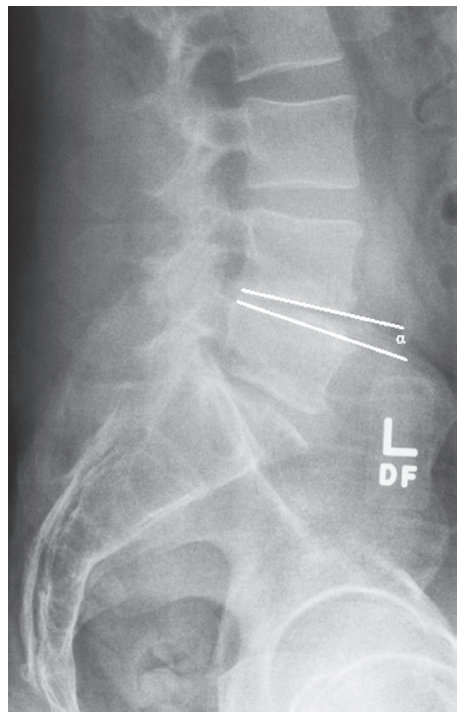

Fig. 4. Preoperative $X$-ray measurement of $L 4-L 5$ disc angle $\left(\alpha=4.7^{\circ}\right)$ in a patient who did not develop pseudarthrosis.

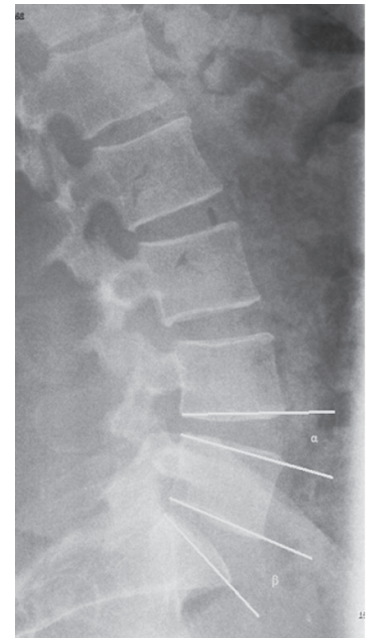

(A)
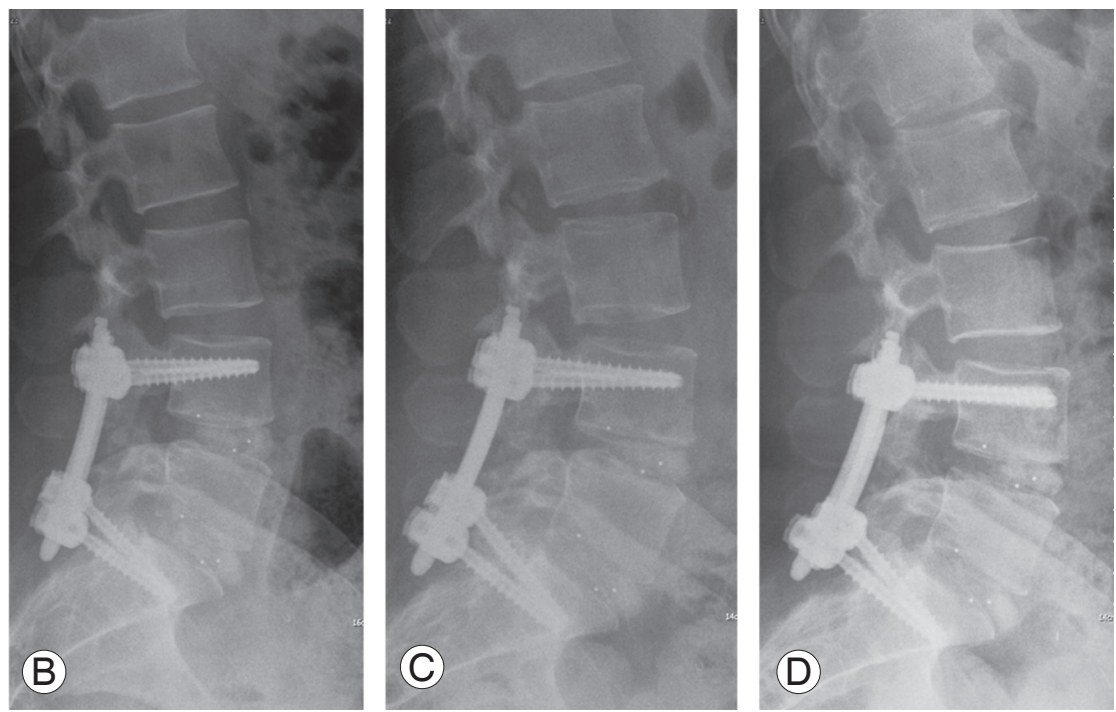

Fig. 5. Preoperative and postoperative $X$-ray measurements of disc angle in the same patient who developed pseudarthrosis. L4-5 $\left(\alpha=14^{\circ}\right)$, L5-S1 $\left(\beta=20^{\circ}\right)$. (A) Preoperative; (B) 6 mo postoperative; (C) 12 mo postoperative; and (D) 18 mo postoperative.

Table 1. Demographics of patients undergoing MIS-TLIF at our institution during 2012-2015

\begin{tabular}{lccc} 
Demographic factor & No pseudarthrosis $(\mathrm{n}=188)$ & Pseudarthrosis $(\mathrm{n}=16)$ & $p$-value \\
\hline Male & $115(61)$ & $10(63)$ & 0.92 \\
\hline Female & $73(39)$ & $6(37)$ & 0.92 \\
\hline Age (yr) & $51(21-81)$ & $47(31-58)$ & 0.311 \\
\hline Follow-up (mo) & $37(32-43)$ & $37(32-42)$ & 1 \\
\hline Smoker & $24(13)$ & $1(6)$ & 0.45 \\
Diabetic & $15(8)$ & $2(13)$ & 0.53 \\
CCl (yr)a) & $1.24(0-5)$ & $0.82(0-1)$ & 0.361 \\
\hline
\end{tabular}

Values are presented as number (\%) or mean (range).

${ }^{a}$ Charlson Comorbidity Index (with age score included). 
Table 2. Operative factors of patients who did not develop pseudarthrosis versus patients who did

\begin{tabular}{lccc} 
Operative factor & No pseudarthrosis $(\mathrm{n}=188)$ & Pseudarthrosis $(\mathrm{n}=16)$ & $p$-value \\
Revision & $9(5)$ & $5(31)$ & 0.0001 \\
\hline Bone morphogenic protein & $49(26)$ & $4(25)$ & 0.93 \\
\hline $\begin{array}{l}\text { No. of levels fused } \\
\text { Level(s) }\end{array}$ & 1.12 & 1.21 & 0.562 \\
\hline L3-L4 & $5(2)$ & 0 & 0.51 \\
L4-L5 & $76(41)$ & $5(31)$ & 0.47 \\
L5-S1 & $67(36)$ & $8(50)$ & 0.25 \\
L4-S1 & $40(21)$ & $3(19)$ & 0.81 \\
\hline
\end{tabular}

Values are presented as number (\%).

Table 3. Radiographic parameters of patients with pseudarthrosis versus patients without pseudarthrosis

\begin{tabular}{lccc}
\multicolumn{1}{c}{ X-ray parameter } & No pseudarthrosis $(\mathrm{n}=152)$ & Pseudarthrosis $(\mathrm{n}=16)$ & $\mathrm{R} 2$ \\
Anterior disc height $(\mathrm{mm})$ & $6(3-15)$ & $17(15-19)$ & 0.79 \\
\hline Posterior disc height $(\mathrm{mm})$ & $4(1-8)$ & $6(3-12)$ & $<0.3$ \\
Anterior/posterior disc height ratio & $1.7(0.5-3.6)$ & $3.6(1.4-5.9)$ & $<0.3$ \\
Disc angle $\left(^{\circ}\right)$ & $4^{\circ}\left(-8^{\circ}-11^{\circ}\right)$ & $16^{\circ}\left(14^{\circ}-18^{\circ}\right)$ & 0.8 \\
\hline
\end{tabular}

Values are presented as mean (range).

at that level. Operative factor data is presented in Table 2. The mean number of levels fused was similar between both groups, with 1.12 levels in the pseudarthrosis cohort and 1.21 levels in the non-pseudarthrosis cohort. Stratification by surgical level did not alter this result. Use of rhBMP-2 also did not affect the rate of revision owing to pseudarthrosis $(p=0.93)$.

Disc angle had the highest $R^{2}(0.8)$, followed by anterior disc height $\left(R^{2}=0.79\right)$. Although posterior disc height and the ratio of anterior to posterior disc height showed a significant relationship with the outcome, the $R^{2}$-values were $<0.3$, thus indicating a less-strong correlation (Table 3). Our overall revision owing to pseudarthrosis rate was $8 \%$. The pseudarthrosis cohort had higher final ODI scores; however, the differences were not statistically significant. In the pseudarthrosis group, the mean ODI scores improved from a mean of $47 \%$ (range, 30\%-65\%) to $26 \%$ (range, $10 \%-32 \%$ ). This was similar to the nonpseudarthrosis cohort, which improved from 45\% (range, $35 \%-64 \%$ ) to $23 \%$ (range, $8 \%-27 \%$ ).

There were no significant differences in the complication rates between the two cohorts during the index procedure. In the pseudarthrosis cohort, there was one complication (6.3\%): late axial low back pain with ra- diculopathy (managed non-operatively). In the nonpseudarthrosis cohort, the complication rate was $8 \%$ and included 11 patients who developed radiculopathy (treated non-operatively), one with postoperative headache caused by incidental durotomy (resolved after 48 hours), one whose cage backed out and who subsequently underwent revision surgery the same day (thus not affecting study results), and two with postoperative radiculopathy caused by misplaced pedicle screws who were also revised and subsequently experienced symptom resolution.

\section{Discussion}

Pseudarthrosis, or nonunion of a fusion, may occur following lumbar spine surgery and can result in expensive and challenging revision surgeries. Various factors, including modifiable patient behaviors, medication use, and systemic diseases, reportedly influence fusion rates [12-15]. Recently, minimally-invasive techniques have been applied to lumbar spine surgery to improve patient outcomes and reduce perioperative morbidity $[19,20]$. Some benefits of MIS-TLIF include decreased postoperative pain, decreased time to ambulation, decreased postoperative narcotic use, decreased intraoperative blood 
loss, decreased wound drainage, shorter hospital course, and quicker return to work [2-6]. However, it is unclear whether applying minimally-invasive techniques to TLIF affects clinically-relevant pseudarthrosis rates.

There have been no reported preoperative radiographic risk factors for revision secondary to treat pseudarthrosis in patients who underwent MIS-TLIF. Sato et al. [21] also recently described a disc height $>10 \mathrm{~mm}$ on preoperative $\mathrm{X}$-ray and MRI as a risk factor for same segment disease (SSD) after surgery in a study population of 163 patients (mean age, 66 years) who underwent either decompression or decompression and fusion with PLIF. SSD was defined as "disk herniation, recurrent stenosis, and others" and it may predispose patients to pseudarthrosis and necessitate re-operation owing to challenges posed by scar tissue, poor tissue healing, insufficient bone graft, insufficient post-operative immobilization, or instability resulting from previous surgery and loss of disc function at the motion segment [17]. Our findings agree with this measurement as a possible risk factor for pseudarthrosis, and we introduce disc angle as another (and potentially stronger) predictor. We also noted the pseudarthrosis cohort had significantly greater disc angles, anterior disc heights, and, to a lesser extent, posterior disc heights and anterior/posterior disc height ratios.

Our findings indicate that discs with more lordosis may be at increased risk. These disc characteristics, which were found to be significant, pose technical challenges to the surgeon, such as incomplete voiding of the disc and undersizing of the interbody cage leading to suboptimal compression by the endplates. These technical difficulties could eventually lead to pseudarthrosis and should be considered during preoperative planning. The L5/S1 and L4/L5 segments can be particularly prone to this scenario. Thus, the authors have modified their approach and operative technique in cases with these disc characteristics, such as by usually using an ALIF approach for the primary fusion. In the authors' experience, ALIF may be helpful because cage undersizing and insufficient disc removal are frequently encountered when using the posterior approach.

Circumferential fusion is the approach of choice for pseudarthrosis revision [22-25]. However, given the results of this study, patients with these disc characteristics may benefit from an anterior approach for the index procedure. In addition to potentially allowing for better exposure to address previously-discussed challenges of a tall or angled disc, an anterior approach also allows the surgeon to take advantage of the superior vascularity and mechanical environment present in the anterior lumbar spine [22-25]. The clinically-relevant pseudarthrosis rate for our MIS-TLIF study population including 204 patients was $8 \%$, similar to those published in lumbar spine surgery [26-29].

Here, revision surgery was the only clinical risk factor for clinically-relevant pseudarthrosis. Of the pseudarthrosis cohort, $31 \%$ were revision cases compared with only $6 \%$ in the non-pseudarthrosis cohort $(p=0.0001)$. Our study findings corroborate with the findings of Sato et al. [21], who reported that almost half of the total number of patients underwent a second surgery owing to SSD. Of the 163 patients, 38 underwent re-operation, and 18 of these 38 operations (47\%) were due to SSD. Further, revision surgery may be a risk factor for pseudarthrosis owing to less autograft being available to the surgeon after removal of facets from the previous laminectomy or discectomy.

Some surgeons choose to augment fusion constructs with rhBMP-2 to promote fusion. Singh et al. [30] reported a 7\% pseudarthrosis rate in 573 patients (mean age, 49 years; range, 26-82 years) treated using minimallyinvasive TLIF augmented with rhBMP-2. The patients received either $4.2 \mathrm{mg}$ or $12 \mathrm{mg}$ of rhBMP-2. In the current study, the use of the extra-small rhBMP-2 kit (2 mg) did not significantly differ between the pseudarthrosis and non-pseudarthrosis groups ( $25 \%$ versus $26 \%, p=0.93$ ). This could be because ultra-low doses of rhBMP-2 were used, or because rhBMP-2 was used on high-risk patients (tobacco users, diabetics) only, or because patients in the pseudarthrosis cohort had disc anatomies that posed a challenge to successful fusion. Ultra-low dose rhBMP-2 was used to avoid complications, such as postoperative radiculitis, heterotopic ossification in the epidural space, and endplate osteolysis/graft subsidence, which are reportedly associated with rhBMP-2 use in the posterior lumbar spine [31]. All patients in the pseudarthrosis cohort were revised with ALIF, and all of them experienced symptom resolution.

Our study has several limitations. Selection or indication bias may have been introduced owing to the singlecenter, retrospective nature of this study. The relatively small sample size of the pseudarthrosis cohort may have resulted in reduced statistical power for detecting significant differences in certain risk factors examined. Also, unmeasured confounding parameters may have influ- 
enced our results. Broader quality of life measures, such as patient activity or satisfaction, were not evaluated. Nevertheless, the authors believe that the observed outcomes are clinically valuable, because this is the first study that evaluates potential radiographic risk factors for pseudarthrosis following MIS-TLIF procedures.

\section{Conclusions}

In summary, pseudarthrosis is not an uncommon complication of lumbar spine surgery. Here, revision surgery and certain radiographic parameters were identified as potential risk factors for clinically-relevant pseudarthrosis following MIS-TLIF. Additionally, the pseudarthrosis rate reported in this study was similar to that published in previous reports. Further, it appeared unaffected by rhBMP-2 usage. Surgeons should consider potentially increased risk for pseudarthrosis in patients having tall or considerably lordosis discs. Further prospective studies with larger sample sizes are warranted to determine the effects of comorbidities, prior surgery, and preoperative disc height and angulation on the development of clinically-relevant pseudarthrosis in patients who undergo MIS-TLIF.

\section{Conflict of Interest}

No potential conflict of interest relevant to this article was reported.

\section{References}

1. Hackenberg L, Halm H, Bullmann V, Vieth V, Schneider M, Liljenqvist U. Transforaminal lumbar interbody fusion: a safe technique with satisfactory three to five year results. Eur Spine J 2005;14:551-8.

2. Wong AP, Smith ZA, Stadler JA 3rd, et al. Minimally invasive transforaminal lumbar interbody fusion (MI-TLIF): surgical technique, long-term 4-year prospective outcomes, and complications compared with an open TLIF cohort. Neurosurg Clin N Am. 2014;25:279-304.

3. Wu WJ, Liang Y, Zhang XK, Cao P, Zheng T. Complications and clinical outcomes of minimally invasive transforaminal lumbar interbody fusion for the treatment of one- or two-level degenerative disc diseases of the lumbar spine in patients older than 65 years. Chin Med J (Engl) 2012;125:2505-10.
4. Peng CW, Yue WM, Poh SY, Yeo W, Tan SB. Clinical and radiological outcomes of minimally invasive versus open transforaminal lumbar interbody fusion. Spine (Phila Pa 1976) 2009;34:1385-9.

5. Perez-Cruet MJ, Hussain NS, White GZ, et al. Quality-of-life outcomes with minimally invasive transforaminal lumbar interbody fusion based on long-term analysis of 304 consecutive patients. Spine (Phila Pa 1976) 2014;39:E191-8.

6. Seng C, Siddiqui MA, Wong KP, et al. Five-year outcomes of minimally invasive versus open transforaminal lumbar interbody fusion: a matched-pair comparison study. Spine (Phila Pa 1976) 2013;38:204955.

7. Adogwa O, Parker SL, Bydon A, Cheng J, McGirt MJ. Comparative effectiveness of minimally invasive versus open transforaminal lumbar interbody fusion: 2-year assessment of narcotic use, return to work, disability, and quality of life. J Spinal Disord Tech 2011;24:479-84.

8. Heggeness MH, Esses SI. Classification of pseudarthroses of the lumbar spine. Spine (Phila Pa 1976) 1991;16(8 Suppl):S449-54.

9. Etminan M, Girardi FP, Khan SN, Cammisa FP Jr. Revision strategies for lumbar pseudarthrosis. Orthop Clin North Am 2002;33:381-92.

10. Heggeness MH, Esses SI, Mody DR. A histologic study of lumbar pseudarthrosis. Spine (Phila Pa 1976) 1993;18:1016-20.

11. Stauffer RN, Coventry MB. Anterior interbody lumbar spine fusion: analysis of Mayo Clinic series. J Bone Joint Surg Am 1972;54:756-68.

12. Carpenter CT, Dietz JW, Leung KY, Hanscom DA, Wagner TA. Repair of a pseudarthrosis of the lumbar spine: a functional outcome study. J Bone Joint Surg Am 1996;78:712-20.

13. Hadley MN, Reddy SV. Smoking and the human vertebral column: a review of the impact of cigarette use on vertebral bone metabolism and spinal fusion. Neurosurgery 1997;41:116-24.

14. Inaoka M, Tada K, Yonenobu K. Problems of posterior lumbar interbody fusion (PLIF) for the rheumatoid spondylitis of the lumbar spine. Arch Orthop Trauma Surg 2002;122:73-9.

15. Sawin PD, Dickman CA, Crawford NR, Melton MS, Bichard WD, Sonntag VK. The effects of dexamethasone on bone fusion in an experimental model of 
posterolateral lumbar spinal arthrodesis. J Neurosurg 2001;94(1 Suppl):76-81.

16. Charlson M, Szatrowski TP, Peterson J, Gold J. Validation of a combined comorbidity index. J Clin Epidemiol 1994;47:1245-51.

17. Lee YP, Sclafani J, Garfin SR. Lumbar pseudarthrosis: diagnosis and treatment. Semin Spine Surg 2011;23:275-81.

18. McAfee PC, Boden SD, Brantigan JW, et al. Symposium: a critical discrepancy-a criteria of successful arthrodesis following interbody spinal fusions. Spine (Phila Pa 1976) 2001;26:320-34.

19. Goldstein CL, Macwan K, Sundararajan K, Rampersaud YR. Comparative outcomes of minimally invasive surgery for posterior lumbar fusion: a systematic review. Clin Orthop Relat Res 2014;472:1727-37.

20. Goz V, Weinreb JH, Schwab F, Lafage V, Errico TJ. Comparison of complications, costs, and length of stay of three different lumbar interbody fusion techniques: an analysis of the Nationwide Inpatient Sample database. Spine J 2014;14:2019-27.

21. Sato S, Yagi M, Machida M, et al. Reoperation rate and risk factors of elective spinal surgery for degenerative spondylolisthesis: minimum 5-year follow-up. Spine J 2015;15:1536-44.

22. Dennis S, Watkins R, Landaker S, Dillin W, Springer D. Comparison of disc space heights after anterior lumbar interbody fusion. Spine (Phila Pa 1976) 1989;14:876-8.

23. Buttermann GR, Glazer PA, Hu SS, Bradford DS. Revision of failed lumbar fusions: a comparison of anterior autograft and allograft. Spine (Phila Pa 1976) 1997;22:2748-55.
24. Albert TJ, Pinto M, Denis F. Management of symptomatic lumbar pseudarthrosis with anteroposterior fusion: a functional and radiographic outcome study. Spine (Phila Pa 1976) 2000;25:123-9.

25. Gertzbein SD, Hollopeter MR, Hall S. Pseudarthrosis of the lumbar spine: outcome after circumferential fusion. Spine (Phila Pa 1976) 1998;23:2352-6.

26. Hutter CG. Posterior intervertebral body fusion: a 25-year study. Clin Orthop Relat Res 1983;(179):8696.

27. Franklin GM, Haug J, Heyer NJ, McKeefrey SP, Picciano JF. Outcome of lumbar fusion in Washington State workers' compensation. Spine (Phila Pa 1976) 1994;19:1897-903.

28. Brantigan JW. Pseudarthrosis rate after allograft posterior lumbar interbody fusion with pedicle screw and plate fixation. Spine (Phila $\mathrm{Pa} 1976$ ) 1994;19:1271-9.

29. Dede O, Thuillier D, Pekmezci M, et al. Revision surgery for lumbar pseudarthrosis. Spine J 2015;15:97782.

30. Singh K, Nandyala SV, Marquez-Lara A, et al. Clinical sequelae after rhBMP-2 use in a minimally invasive transforaminal lumbar interbody fusion. Spine J 2013;13:1118-25.

31. Chrastil J, Low JB, Whang PG, Patel AA. Complications associated with the use of the recombinant human bone morphogenetic proteins for posterior interbody fusions of the lumbar spine. Spine (Phila Pa 1976) 2013;38:E1020-7. 